\title{
Momentum and Heat Transfer in MHD Axisymmetric Stagnation- Point Flow over a Shrinking Sheet
}

\author{
T. R. Mahapatra ${ }^{1 \dagger}$ and S. K. Nandy ${ }^{2}$ \\ ${ }^{I}$ Department of Mathematics, Visva-Bharati, Santiniketan-731 235, India \\ ${ }^{2}$ Department of Mathematics, A.K.P.C Mahavidyalaya, Bengai, Hooghly-712 611, India \\ †Corresponding Author Email: trmahapatra@yahoo.com
}

(Received January 1, 2011; accepted October 9, 2011)

\begin{abstract}
In this paper we present a mathematical analysis for the magneto hydrodynamic (MHD) axi-symmetric stagnation-point flow and heat transfer over a shrinking sheet which shrinks axi-symmetrically in its own plane. The governing partial differential equations along with the boundary conditions are first cast into a dimensionless form and then these equations are solved numerically by shooting technique. Thermal conductivity is assumed to vary linearly with the temperature. Temperature profiles are obtained for two different types of heating process namely (i) the sheet with prescribed surface temperature (PST) and (ii) the sheet with prescribed surface heat flux (PHF). The effects of various physical parameters on the flow and heat transfer characteristics are presented graphically and discussed.
\end{abstract}

Keywords: Magnetohydrodynamic, Axi-symmetric stagnation-point flow, Temperature dependent thermal conductivity, Heat transfer, Shrinking sheet.

\section{NOMENCLATURE}

a positive constant proportional to the free stream straining velocity

A positive constant

$\mathrm{B}_{0}$ uniform magnetic field acting transverse to the sheet

c proportionality constant of the velocity of the sheet

$c_{p} \quad$ specific heat of the fluid at constant pressure

D positive constant

$\mathrm{E}_{\mathrm{c}} \quad$ Eckert number

1 dimensional distance from the stretching/shrinking origin

L non-dimensional distance from the stretching/shrinking origin

$l_{1} \quad$ characteristic length

M magnetic parameter

$\mathrm{p}$ pressure of the fluid

Pr Prandtl number

$\mathrm{q}_{\mathrm{w}}$ heat flux at the surface

$\mathrm{R}$ reciprocal of the dimensionless distance along the sheet

$\mathrm{T}$ temperature of the fluid

$\mathrm{T}_{\mathrm{w}}$ wall temperature

$\mathrm{T}_{\infty}$ temperature of the free stream fluid
$\Delta \mathrm{T}$ difference between wall temperature and free stream temperature of the fluid

$\mathrm{u}, \mathrm{u}^{*}$ dimensional, non-dimensional velocity component in the $\mathrm{x}$ direction

$\mathrm{U}$ free stream velocity in $\mathrm{x}$ direction

$\mathrm{w}, \mathrm{w}^{*}$ dimensional, non-dimensional velocity component in the $\mathrm{z}$ direction

$\mathrm{W}$ free stream velocity in $\mathrm{z}$ direction

$\mathrm{x}$ dimensional distance along the sheet

$\mathrm{z}$ dimensional distance normal to the sheet

$\alpha \quad$ ratio of the shrinking and free stream velocities

$\varepsilon \quad$ constant that appears due to temperature dependent thermal conductivity

$\kappa \quad$ thermal conductivity of the fluid

$\kappa_{\infty} \quad$ thermal conductivity of the fluid in free stream

$\theta$ dimensionless temperature

$\xi \quad$ dimensionless distance along the sheet

$\eta \quad$ dimensionless distance normal to the sheet

$\tau$ dimensionless surface shear stress

$\rho$ density of the fluid

$\mu \quad$ dynamic coefficient of viscosity of the fluid

$v \quad$ kinematic viscosity of the fluid

$\sigma \quad$ electrical conductivity of the fluid 


\section{INTRODUCTION}

The study of boundary layer flow over a stretching /shrinking sheet is a subject of great interest due to its various applications in designing cooling system which includes liquid metals, MHD generators, accelerators, pumps and flow meters. Furthermore, the continuous surface heat and mass transfer problems are many practical applications in electro-chemistry and polymer processing. Many chemical engineering processes, like metallurgical and polymer extrusion involve cooling of a molten liquid being stretched into a cooling system. The fluid mechanical properties of the penultimate product depend mainly on the process of stretching and on the rate of cooling.

Hydromagnetic behavior of boundary layer flow over a moving surface in the presence of transverse magnetic field is a basic and important problem in magnetohydrodynamic (MHD). The MHD flow and heat transfer for a viscous fluid over a stretching/shrinking sheet has enormous applications in many engineering problems such as plasma studies, petroleum industries, geothermal energy extractions, the boundary layer flow control in the field of aerodynamic and many others.

Stagnation-point flow is a topic of significance in fluid mechanics, in the sense that it appears in virtually all flow fields of science and engineering. In some cases, flow is stagnated by a solid wall, while in others a free stagnationpoint or a line exists interior of the fluid domain. Hiemenz (1911) was the first to solve the two-dimensional stagnationpoint flow problem using a similarity transformation and the axi-symmetric three dimensional stagnation flow problem was studied by Homman (1936). Later the problem of axisymmetric stagnation flow over a moving surface is extended in numerous ways to include various physical effects. The results of these studies are of great technical importance, such as in the prediction of skin friction as well as heat/mass transfer near stagnation regions of bodies in high speed flows and also in the design of thrust bearing and radial diffusers, drag reduction, transpiration cooling and thermal oil recovery.

Crane (1970) studied the steady two-dimensional boundary layer flow of an incompressible viscous fluid caused by the stretching of an elastic flat surface which moves in its own plane with a velocity varying linearly with the distance from a fixed point. Heat transfer in the above flow maintained at constant as well as variable wall temperature was investigated by Gupta and Gupta (1977) and also by Carragher and Crane (1982). Wang (1984) analyzed the steady three dimensional flow of a viscous fluid over a plane surface which is stretched in its own plane in two perpendicular directions. The flow caused by the axisymmetric stretching of the surface was also investigated by him. Pavlov (1974) gave an exact similarity solution of the MHD boundary layer equations for the steady twodimensional flow of an electrically conducting incompressible fluid due to the stretching of an elastic surface in the presence of a uniform transverse magnetic field. Heat transfer in the above flow with suction or blowing was analyzed by Chakrabarti and Gupta (1979).
Chiam (1994) investigated the steady axisymmetric stagnation-point flow of a viscous fluid over an elastic surface which is stretched axisymetrically. Mahapatra and Gupta (2002) investigated flow and heat transfer in twodimensional orthogonal stagnation-point flow of an incompressible viscous fluid towards a stretching surface. They found that the structure of the boundary layer depends on the ratio of the velocity of the frictionless potential flow to that of the stretching surface. The corresponding problem of axisymmetric stagnation-point flow of an incompressible viscous fluid towards a stretching surface was also analyzed by Mahapatra and Gupta (2003). Axisymmetric stagnationpoint flow towards a stretching surface in the presence of a uniform transverse magnetic field with heat generation was investigated by Attia (2007). The similarity solution for an unsteady MHD stagnation-point flow of a three dimensional porous body with heat and mass transfer was investigated by Chamkha and Ahamed (2011).

The boundary layer flow due to a shrinking sheet has attracted considerable interest recently. From consideration of continuity, Crane's (1970) stretching sheet solution induces a far field suction towards the sheet, while flow over a shrinking sheet would give rise to a velocity away from the sheet. From a physical point of view, vorticity generated at the shrinking sheet is not confined within a boundary layer and a steady flow is not possible unless adequate suction is applied at the surface. For this type of shrinking flow, it is essentially a backward flow as discussed by Goldstein (1965). For a backward flow configuration, the fluid losses any memory of the perturbation introduced by the sheet. As a result, the flow induced by the shrinking sheet shows quite distinct physical phenomena from the forward stretching case.

Miklavcic and Wang (2006) investigated both twodimensional and axisymmetric viscous flow induced by a shrinking sheet in the presence of uniform suction. The above shrinking sheet problem was extended to power-law surface velocity by Fang (2008). Fang and Zhang (2009) gave an exact solution of MHD boundary layer equations in closed analytical form for flow of an electrically conducting fluid over a shrinking sheet in the presence of suction at the surface, the flow being permeated by a uniform transverse magnetic field. Steady two-dimensional and axisymmetric stagnation-point flow with heat transfer on a shrinking sheet was investigated by Wang (2008) and the same problem was solved analyticall by Rahimpour et al. (2008). Recently, Mahapatra et al. (2011) studied steady two-dimensional MHD stagnation-point flow of an electrically conducting incompressible viscous fluid over a shrinking sheet, the flow being permeated by a uniform transverse magnetic field. Note that with an added stagnation-point flow to contain the vorticity, similarity solution is possible even in the absence of suction at the surface.

All the above investigators restrict their analyses to MHD flow and heat transfer over a stretching/shrinking sheet with constant thermal conductivity. It was observed by Savvas et al. (1994) that for liquid metals, the thermal conductivity varies linearly with temperature in the range $0-400^{\circ} \mathrm{F}$. With this assumption, different authors solve the heat transfer 
problem under various physical conditions [see Prasad et al. (2009), Prasad and Vajravelu (2009), Sharma and Singh (2009)]. Hence we assume that the thermal conductivity is a linear function of the temperature.

To the best of our knowledge, no investigation is made for the MHD axi-symmetric stagnation-point flow of an incompressible viscous fluid over a shrinking sheet. In this paper, we study the axi-symmetric stagnation-point flow and heat transfer phenomenon over a shrinking surface, in the presence of uniform transverse magnetic field, taking into the account of variable thermal conductivity. We consider two different cases on non-isothermal boundary conditions namely, (i) surface with prescribed surface temperature (PST case) and (ii) surface with prescribed wall heat flux (PHF case)

\section{Flow Analysis}

Consider the steady axisymmetric stagnation-point flow of an electrically conducting incompressible viscous fluid towards a surface which is shrunk axisymmetrically with a velocity proportional to the distance from the shrinking origin. Due to possible non-alignment, it is more appropriate to use Cartesian axes instead of cylindrical axes (see Wang(2008)). Let ( $\mathrm{u}, \mathrm{v}, \mathrm{w})$ be the velocity components in the Cartesian coordinates $(\mathrm{x}, \mathrm{y}, \mathrm{z})$, respectively. On the sheet, the velocities are $\mathrm{u}=\mathrm{c}(\mathrm{x}+\mathrm{l}), \mathrm{v}=\mathrm{cy}, \mathrm{w}=0$, where $\mathrm{c}(<0)$ is the shrinking rate (stretching rate if $c>0$ ) and -1 is the location of the shrinking (stretching) origin. Here shrinking of the sheet is along the negative direction of $\mathrm{x}$-axis. Notice that the stretching axis and the stagnation-point flow are not, in general, aligned $(1 \neq 0)$. The velocity components at infinity are given by $U=a x, V=a y$ and $W=-2 a z$ where $a(>0)$ is the strength of the stagnation-point flow. A uniform magnetic field $\mathrm{B}_{0}$ is applied in a direction normal to the surface i.e., parallel to Z-axis. The flow configuration is shown in Fig 1.

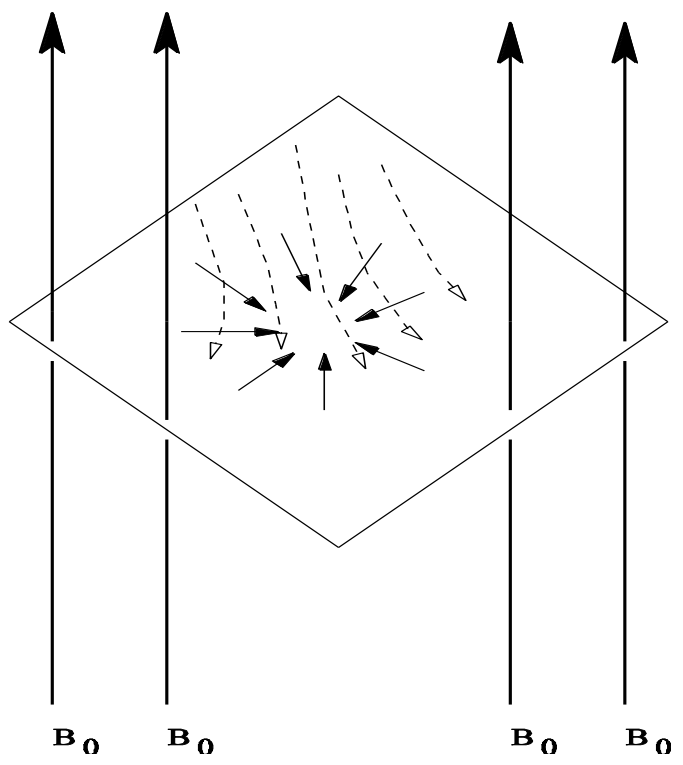

Fig 1. A sketch of the physical problem.
The governing equations of continuity and momentum under the influence of externally imposed transverse magnetic field Bansal (1994) in the boundary layer are

$$
\begin{aligned}
& \frac{\partial u}{\partial x}+\frac{\partial u}{\partial y}+\frac{\partial w}{\partial z}=0 \\
& u \frac{\partial u}{\partial x}+w \frac{\partial u}{\partial z}=-\frac{1}{\rho} \frac{\partial p}{\partial x}+v \frac{\partial^{2} u}{\partial z^{2}}-\frac{\sigma B_{0}^{2}}{\rho} u
\end{aligned}
$$

where $\rho, v, \sigma$ and $\mathrm{p}$ denote the density, kinematic viscosity, electrical conductivity and the pressure of the fluid. The last term in Eq. (2) is due to the Lorentz force. In writing Eq. (2), we have neglected the induced magnetic field since the magnetic Reynolds number $R_{M}$ for the flow is assumed to be very small. This assumption is justified for flow of electrically conducting fluids such as liquid metals e.g., mercury, liquid sodium etc. Shercliff (1965).

The pressure gradient $\frac{\partial p}{\partial x}$ can now be obtained from Eq. (2) in the free stream as

$$
-\frac{1}{\rho} \frac{\partial p}{\partial x}=U \frac{d U}{d x}+\frac{\sigma B_{0}^{2}}{\rho} U
$$

Eliminating $\frac{\partial p}{\partial x}$ from Eqs. (2) and (3), we get

$u \frac{\partial u}{\partial x}+w \frac{\partial u}{\partial z}=U \frac{d U}{d x}+v \frac{\partial^{2} u}{\partial z^{2}}+\frac{\sigma B_{0}{ }^{2}}{\rho}(U-u)$

The boundary conditions for the above flow situation are

$u=c(x+l), v=c y, w=0$ at $z=0$
$u \rightarrow U(x)=a x, \quad$ as $z \rightarrow \infty$

where $\mathrm{a}(>0)$ is a constant.

Following transformations are introduced in accordance with Wang (2008):

$$
u=\operatorname{ax} F^{\prime}(\eta)+\operatorname{clh}(\eta), v=a y F^{\prime}(\eta), w=-2 \sqrt{a v} F(\eta)
$$

where $\eta$ is the dimensionless similarity variable given by

$\eta=z\left(\frac{a}{v}\right)^{1 / 2}$,

and a prime denotes differentiation with respect to $\eta$. With $u$ and $\mathrm{w}$ given by Eq. (7), the equation of continuity (1) is identically satisfied. Substituting Eqs. (7) and (8) in Eq. (4) and equating the coefficients of $x^{0}$ and $x^{1}$, we obtain the following non-linear differential equations:

$$
\begin{aligned}
& F^{\prime \prime}+2 F F^{\prime \prime}-F^{\prime 2}+1+M^{2}\left(1-F^{\prime}\right)=0, \\
& h^{\prime \prime}+2 F h^{\prime}-h F^{\prime}-M^{2} h=0 .
\end{aligned}
$$

In Eqs. (9) and (10), the constant $M=\left(\sigma B_{0}{ }^{2} / a \rho\right)^{1 / 2}$ is the magnetic parameter characterizing the strength of the imposed magnetic field. The appropriate boundary 
conditions for $F(\eta)$ and $h(\eta)$ are obtained from Eqs. (5)-(8) as

$$
\begin{aligned}
& F(0)=0, F^{\prime}(0)=\frac{c}{a}=\alpha, F^{\prime}(\infty)=1, \\
& h(0)=1, \quad h(\infty)=0 .
\end{aligned}
$$

The dimensionless velocity components can be written from

Eq. (7) as $u^{*}=\frac{u}{\sqrt{a v}}=\xi F^{\prime}(\eta)+\alpha \operatorname{Lh}(\eta)$

$w^{*}=\frac{w}{\sqrt{a v}}=-2 F(\eta)$,

where

$\xi=x \sqrt{\frac{a}{v}}$ and $L=l \sqrt{\frac{a}{v}}$.

The dimensionless wall shear stress $\tau$ is given by

$$
\tau=\xi F^{\prime \prime}(0)+\alpha L h^{\prime}(0) \text {. }
$$

\section{Heat Transfer}

The energy equation for a fluid with variable thermal conductivity in the presence of viscous and ohomic dissipations for the above flow is given by [Chiam (1998)]

$$
\begin{aligned}
& \rho c_{p}\left(u \frac{\partial T}{\partial x}+w \frac{\partial T}{\partial z}\right)=\frac{\partial}{\partial z}\left(\kappa(T) \frac{\partial T}{\partial z}\right) \\
& +\mu\left(\frac{\partial u}{\partial z}\right)^{2}+\sigma B_{0}^{2}(u-U)^{2},
\end{aligned}
$$

where $c_{p}$ is the specific heat at constant pressure, $T$ is the temperature of the fluid and $\kappa(\mathrm{T})$ is the temperature dependent thermal conductivity. We consider the temperature dependent thermal conductivity in the following form [Chiam (1998)]

$$
\kappa(T)=\kappa_{\infty}\left[1+\frac{\varepsilon}{\Delta T}\left(T-T_{\infty}\right)\right],
$$

where $\kappa_{\infty}$ is the conductivity of the fluid far away from the sheet, $\Delta \mathrm{T}=\mathrm{T}_{\mathrm{w}}-\mathrm{T}_{\infty}, \mathrm{T}_{\mathrm{w}}$ is the sheet temperature and $\mathrm{T}_{\infty}$ is the free stream temperature and $\varepsilon$ is a small parameter. Substituting Eq. (18) into Eq. (17), we get

$$
\begin{aligned}
& \rho c_{p} u \frac{\partial T}{\partial x}+\left(\rho c_{p} w-\frac{\kappa_{\infty} \varepsilon}{\Delta T} \frac{\partial T}{\partial z}\right) \frac{\partial T}{\partial z} \\
& =\kappa(T) \frac{\partial^{2} T}{\partial z^{2}}+\mu\left(\frac{\partial u}{\partial z}\right)^{2}+\sigma B_{0}{ }^{2}(u-U)^{2}
\end{aligned}
$$

The thermal boundary conditions depend on the type of heating process under consideration. Here, we consider two different heating processes, namely (i) Prescribed Surface Temperature and (ii) Prescribed Wall Heat Flux.

\section{Case 1: Prescribed Surface Temperature (PST)}

We assume that the prescribed wall temperature is a quadratic function of $r$ given by

$$
\begin{aligned}
& T=T_{w}=T_{\infty}+A\left(\frac{x}{l_{1}}\right)^{2} \text { at } z=0 \\
& T \rightarrow T_{\infty} \quad \text { as } z \rightarrow \infty
\end{aligned}
$$

where $T_{w}$ is the variable wall temperature, $A$ is a constant and $l_{1}$ is a characteristic length. We take the dimensionless temperature $\theta$ as

$\theta=\frac{T-T_{\infty}}{T_{w}-T_{\infty}}$,

where $\mathrm{T}_{\mathrm{w}}-\mathrm{T}_{\infty}=\mathrm{A}\left(\mathrm{x} / \mathrm{l}_{1}\right)^{2}$. Substituting Eqs. (20), (21) into Eq. (19), we get

$$
\begin{aligned}
& (1+\varepsilon \theta) \theta^{\prime \prime}+\varepsilon \theta^{\prime 2}+\operatorname{Pr}\left[2 F \theta^{\prime}\right. \\
& -2\left(F^{\prime}+\alpha R L h\right) \theta+E_{c}\left(F^{\prime \prime}+\alpha R L h^{\prime}\right)^{2} \\
& \left.+E_{c} M^{2}\left(F^{\prime}-1+\alpha R L h\right)^{2}\right]=0
\end{aligned}
$$

where a prime denotes differentiation with respect to $\eta$ and $\operatorname{Pr}$ and $\mathrm{E}_{\mathrm{c}}$ denote the Prandtl number and the Eckert number, respectively. They are defined as follows:

$\operatorname{Pr}=\frac{\mu c p}{\kappa_{\infty}}, E_{c}=\frac{a^{2} l_{1}^{2}}{c_{p} A}, R=\frac{1}{\xi}$

The boundary conditions are

$\theta(0)=1, \quad \theta(\infty)=0$.

\section{Case 2: Prescribed Wall Heat Flux (PHF)}

The heat flux $\left(\mathrm{q}_{\mathrm{w}}\right)$ at the surface is assumed to vary as the square of the distance as follows:

$$
\begin{aligned}
& -\kappa_{\infty} \frac{\partial T}{\partial z}=q_{w}=D\left(\frac{x}{l_{1}}\right)^{2} \text { at } z=0 \\
& T \rightarrow T_{\infty} \quad \text { as } z \rightarrow \infty
\end{aligned}
$$

where $\mathrm{D}$ is a constant. Here we let

$$
T-T_{\infty}=\frac{D}{\kappa_{\infty}} \sqrt{\frac{v}{a}}\left(\frac{x}{l_{1}}\right)^{2} g(\eta)
$$

The energy Eq. (19) is transformed into the equation

$$
\begin{aligned}
& (1+\varepsilon g) g^{\prime \prime}+\varepsilon g^{\prime 2}+\operatorname{Pr}\left[2 F g^{\prime}\right. \\
& -2\left(F^{\prime}+\alpha R L h\right) g+E_{c}\left(F^{\prime \prime}+\alpha R L h^{\prime}\right)^{2} \\
& \left.+E_{c} M^{2}\left(F^{\prime}-1+\alpha R L h\right)^{2}\right]=0
\end{aligned}
$$

subject to the boundary conditions

$$
g^{\prime}(0)=-1, \quad g(\infty)=0 \text {. }
$$

where a prime denotes differentiation with respect to $\eta$ and the Eckert number $\quad E_{c}=\frac{\kappa_{\infty} a^{2} l_{1}^{2} \sqrt{a / v}}{D c_{p}}$. It is to be noted 
that Eq. (27) is exactly the same form as Eq. (22) but the first boundary condition is now different.

\section{NUMERICAL SOLUTION}

The transformed momentum Eqs. (9) and (10) subject to the boundary conditions (11) and (12) are solved numerically by shooting technique for different values of the physical parameters. First, Eq. (9) is written as a system of three first order differential equations, which are solved by means of a standard fourth-order Runge-Kutta integration technique. Then a Newton iteration procedure is employed to assure quadratic convergence of the iterations required to satisfy the boundary conditions $F^{\prime}(\infty)=1$. Using the falues of $F(\eta)$ obtained from Eqs. (9) and (11), we get $h(\eta)$ by solving Eq. (10) together with the boundary conditions (12) numerically by the same technique as described above.

Using the numerical values of $F(\eta)$ and $h(\eta)$ from the solutions for the velocity distribution in section 2, Eqs (22) and (24) for the PST case and Eqs. (27) and (28) for the PHF case are solved to obtain $\theta(\eta)$ numerically by employing a shooting technique.

\section{Result ANd CONCLUSION}

In order to assess the accuracy of the numerical method, we have compared the local skin friction coefficients $F$ " $(0)$, $h^{\prime}(0)$ and wall temperature gradient $-\theta^{\prime}(0)$ for constant surface temperature for different values of $\alpha$ with the previously published data (Wang 2008; Rahimpour et al. 2008) available in the literature. Wang (2008) numerically solved the stagnation point flow problem over a shrinking sheet in the absence of magnetic field and Rahimpour et al. (2008) solved the same problem analytically. The comparisons are shown in Tables 1 and 2, which show a favorable agreement, and thus give confidence that the numerical results obtained are accurate.

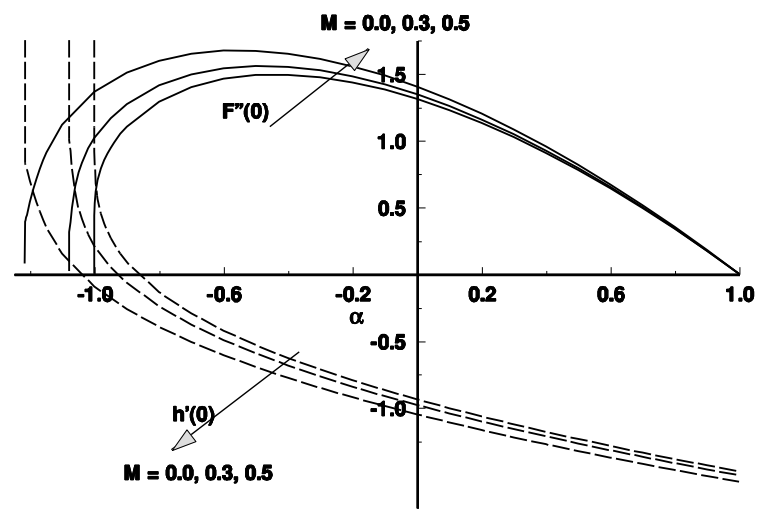

Fig. 2. Trajectories of $F^{\prime \prime}(0)$ and h'(0) for different values of the magnetic parameter $M$.
Fig. 2 shows the trajectories of the values of F" $(0)$ and h'(0) for different values of the magnetic parameter $M$. In this figure, the trajectories for F" (0) are represented by solid lines while those for $h^{\prime}(0)$ are shown as dashed lines. Our numerical results reveal that in the absence of the magnetic parameter (i.e., $M=0$ ), the solutions of Eqs. (9) and (10) for $F(\eta)$ and $h(\eta)$ satisfying the boundary conditions (11) and (12) respectively, are unique for $\alpha \geq-1$ and no similarity solution exists for $\alpha<-1$. The above result agree well with those of Wang (2008). The novel result that emerges from the analysis is that as $M$ increases, the range of $\alpha$ where similarity solutions exist gradually increases. When $\alpha=1$, we find that $F^{\prime \prime}(0)=0$ because $F(\eta)=\eta$ is the solution of Eq. (9) subject to the boundary conditions (11). The figure reveals that for the flow over a shrinking sheet, $F^{\prime \prime}(0) \geq 0$ and for a given value of $\alpha, F^{\prime \prime}(0)$ increases with increase in $M$. The trajectory for h' $(0)$ crosses the $\alpha$-axis in the case of flow over a shrinking sheet but it does not cross the $\alpha$-axis for flow over a stretching sheet. For a given value of the magnetic parameter $M$, the magnitude of $h^{\prime}(0)$ decreases with increase in $\alpha$. Also for a given value of $\alpha,\left|h^{\prime}(0)\right|$ increases with increase in $\mathrm{M}$.

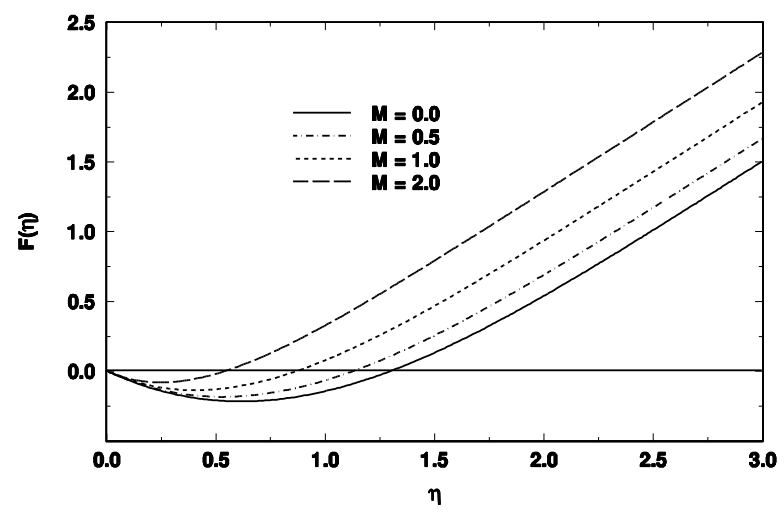

Fig. 3. Variation of $F(\eta)$ with $\eta$ for $\alpha=-0.75$ (shrinking) for several values of magnetic parameter $M$.

Fig. 3 shows the variation of the vertical velocity component $F(\eta)$ with $\eta$ for different values of the magnetic parameter $\mathrm{M}$. It is interesting to note that for shrinking at the surface $(\alpha<0)$, the function $F(\eta)$ is negative near the shrinking sheet, showing regions of reverse cellular flow. The figure indicates that as $\mathrm{M}$ increases, the region of reverse cellular flow decreases. 
T. R. Mahapatra et al. / JAFM, Vol. 6, No. 1, pp. 121-129, 2013.

Table 1 Comparison of the values of $F^{\prime \prime}(0)$ and $h^{\prime}(0)$ when the magnetic parameter $M=0$

\begin{tabular}{|c|c|c|c|c|c|c|}
\hline & \multicolumn{3}{|c|}{$F^{\prime \prime}{ }^{\prime}(0)$} & \multicolumn{3}{c|}{$h^{\prime}(0)$} \\
\hline$\alpha$ & Wang(2008) & Rahimpour et al. (2008) & Present study & Wang(2008) & Rahimpour et al. (2008) & Present study \\
\hline-0.95 & 0.9469 & 0.946815 & 0.946893 & 0.26845 & 0.268450 & 0.268457 \\
\hline-0.75 & 1.35284 & 1.352850 & 1.352841 & -0.22079 & -0.220789 & -0.220795 \\
\hline-0.50 & 1.49001 & 1.490004 & 1.490013 & -0.53237 & -0.532371 & -0.5 .32374 \\
\hline-0.25 & 1.45664 & 1.456599 & 1.456641 & -0.75639 & -0.756390 & -0.756380 \\
\hline 0.0 & 1.31393 & 1.311938 & 1.311942 & -0.93873 & -0.938732 & -0.938731 \\
\hline 0.1 & 1.22911 & 1.229113 & 1.229111 & -1.00400 & -1.004026 & -1.004031 \\
\hline 0.5 & 0.78032 & 0.780323 & 0.780327 & -1.23550 & -1.235451 & -1.235460 \\
\hline 1.0 & 0.0 & 0.0 & 0.0 & -1.47930 & -1.479337 & -1.479341 \\
\hline 2.0 & -2.13107 & -2.131069 & -2.131068 & -1.88000 & -1.879949 & -1.879956 \\
\hline 5.0 & $-11 . .8022$ & $-11 . .802214$ & -11.802202 & -2.76170 & -2.761724 & -2.761702 \\
\hline
\end{tabular}

Table 2 Comparison of the values of $-\theta^{\prime}(0)$ in the case of constant surface temperature

\begin{tabular}{|c|c|c|c|c|}
\hline & \multicolumn{2}{|c|}{$\operatorname{Pr}=0.7$} & \multicolumn{2}{c|}{$\operatorname{Pr}=7.0$} \\
\hline$\alpha$ & Rahimpour et al. (2008) & Present study & Rahimpour et al. (2008) & Present study \\
\hline-0.25 & 0.57485972 & 0.57483044 & 1.05649153 & 1.05647470 \\
\hline-0.50 & 0.46709271 & 0.46706751 & 0.51204057 & 0.51203228 \\
\hline-0.75 & 0.32600021 & 0.32591741 & 0.07191553 & 0.07190594 \\
\hline-0.95 & 0.13688695 & 0.13684707 & 0.00003548 & 0.00003674 \\
\hline 0.0 & 0.66540000 & 0.66537890 & 1.54570000 & 1.54578755 \\
\hline 0.5 & 0.81676798 & 0.81678773 & 2.34510969 & 2.34514549 \\
\hline 1.0 & 0.94406979 & 0.94407350 & 2.98541099 & 2.98541957 \\
\hline
\end{tabular}

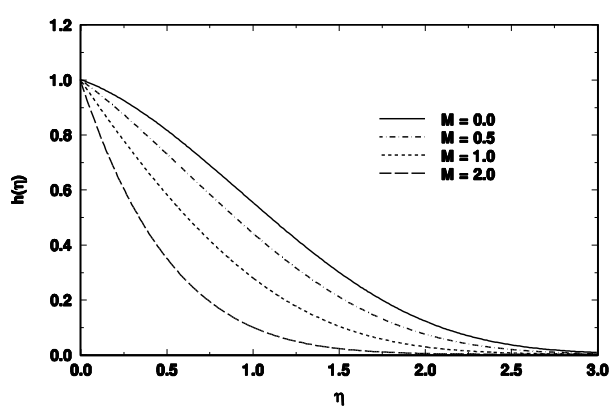

Fig. 4. Variation of $h(\eta)$ with $\eta$ for $\alpha=-0.75$ (shrinking) for several values of magnetic parameter $M$.

Fig. 4 shows the variation of the non-alignment function $h(\eta)$ with $\eta$ for different values of $M$ for a fixed value of $\alpha$ $(=-0.75)$. It is seen that for shrinking at the sheet $h(\eta)$ decreases as $\mathbf{M}$ increase. Thus we can conclude that for shrinking at the sheet, the effect of non-alignment becomes less pronounced with increasing M. Fig. 5 shows the variation of the horizontal velocity component $u^{*}(\xi, \eta)$ with $\eta$ for several values of $M$ with fixed values of $\alpha$, $L$ and $\xi$. It is seen that the horizontal component of velocity increases with increase in $\mathrm{M}$.

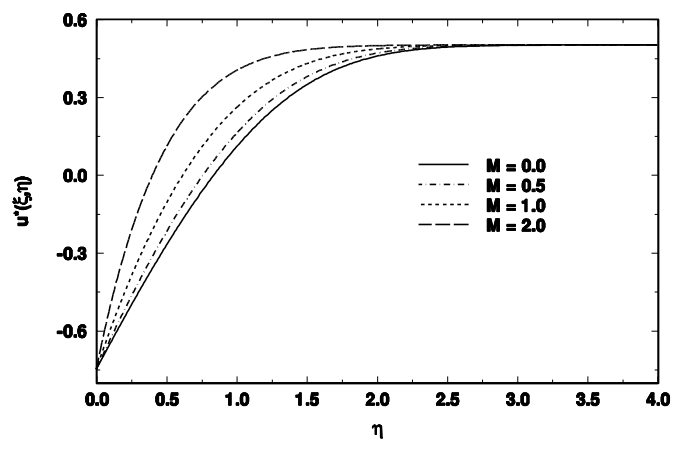

Fig. 5. Variation of $u^{*}(\xi, \eta)$ with $\eta$ for several values of $M$ with $\alpha=-0.5, L=1.0$ and $\xi=0.5$.

The effect of the magnetic parameter $\mathrm{M}$ on the temperature profile in the presence of the variable thermal conductivity parameter $\varepsilon$ (keeping other parameters fixed) for both PST and PHF cases are displayed in Figs. 6 and 7, respectively. It is observed that the effect of $M$ is to decrease the temperature profiles for both PST and PHF cases. From a physical point of view, this follows from the fact that the extent of the reverse cellular flow above the sheet decreases with increase in $\mathrm{M}$. This is a consequence of the fact that the temperature field given by Eq. (17) is influenced by the advection of the fluid velocity above the sheet. 


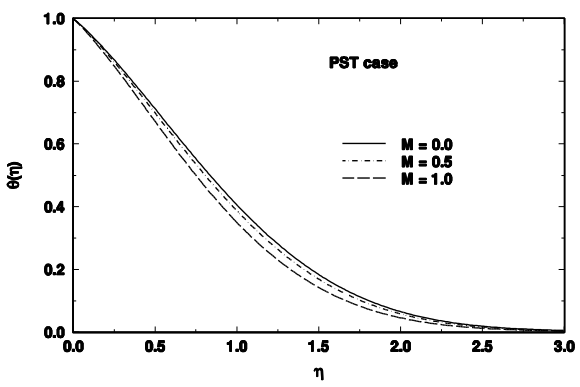

Fig. 6. Variation of $\theta(\eta)$ with $\eta$ for several values of the magnetic parameter $M$ with $\alpha=-0.5, P r=0.72, E_{c}=0.1, \varepsilon=0.1$, $L=1.0, R=10.0 .(P S T$ case $)$

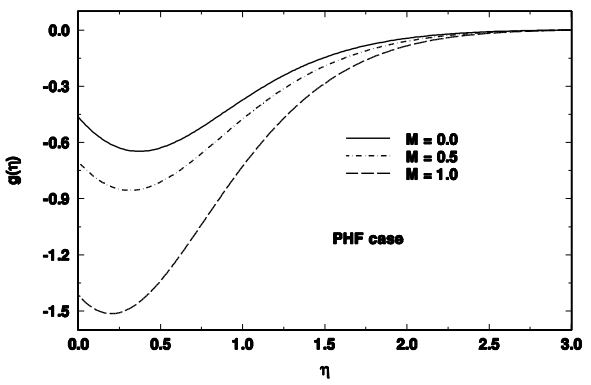

Fig. 7. Variation of $g(\eta)$ with $\eta$ for several values of the magnetic parameter $M$ with $\alpha=-0.5, \operatorname{Pr}=0.72, E_{c}=0.1, \varepsilon=0.1$, $L=1.0, R=10.0$. (PHF case)

Figures 8 and 9 exhibit the temperature distribution for different values of the thermal conductivity parameter $\varepsilon$ (keeping other parameters fixed) in PST and PHF cases, respectively. The effect of variable thermal conductivity parameter $\varepsilon$ is to increase the temperature profile with the increase of $\varepsilon$, which in turn increases the thermal boundary layer thickness for both PST and PHF cases.

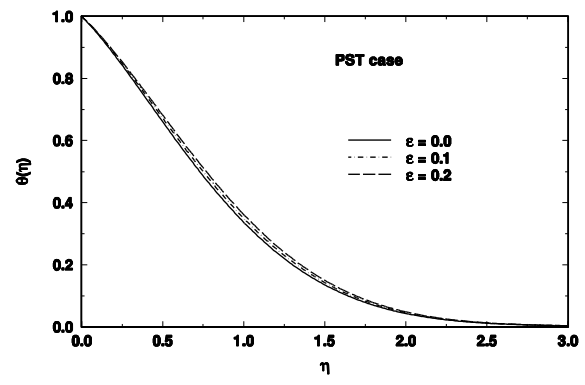

Fig. 8. Variation of $\theta(\eta)$ with $\eta$ for several values of $\varepsilon$ with $\alpha=-0.5, \operatorname{Pr}=0.72, E_{c}=0.1, M=1.0, L=1.0, R=10.0$. (PST case)

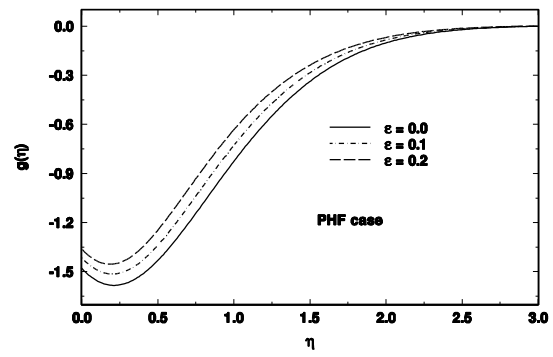

Fig. 9. Variation of $\mathrm{g}(\eta)$ with $\eta$ for several values of $\varepsilon$ with $\alpha=-0.5, \operatorname{Pr}=0.72, E_{c}=0.1, M=1.0, L=1.0, R=10.0$. $(P H F$

case)

The heat transfer phenomena is usually analyzed from the numerical values of the physical parameters viz. (i) wall temperature gradient $-\theta^{\prime}(0)$ in PST case and (ii) wall temperature $\theta(0)$ in PHF case and these results are recorded in Tables 3 and 4 . It is observed that the effect of the magnetic parameter $M$ is to increase the magnitude of the wall temperature gradient $\left|-\theta^{\prime}(0)\right|$ in PST case and the magnitude of wall temperature $|\theta(0)|$ in PHF case. When the thermal conductivity parameter $\varepsilon$ increases, the magnitude of wall temperature $|\theta(0)|$ in PHF case decreases. But upto certain value of $\alpha(<0)$, the magnitude of the wall temperature gradient $\left|-\theta^{\prime}(0)\right|$ in PST case decreases as $\varepsilon$ increases but beyond this value of $\alpha,\left|-\theta^{\prime}(0)\right|$ increases with the increase in $\varepsilon$. Also as $|\alpha|$ increases, $\left|-\theta^{\prime}(0)\right|$ for PST case and $|\theta(0)|$ for PHF case decrease.

Table 3 Wall temperature gradient $-\theta^{\prime}(0)$ for the PST case taking $\operatorname{Pr}=0.72, E_{c}=0.1, R=10.0$ and $L=1.0$

\begin{tabular}{|c|c|c|c|c|}
\hline$\varepsilon$ & $\alpha$ & $\mathrm{M}=0.0$ & $\mathrm{M}=0.5$ & $\mathrm{M}=1.0$ \\
\hline \multirow{3}{*}{0.0} & -0.25 & 0.705507 & 0.715416 & 0.735146 \\
\cline { 2 - 5 } & -0.50 & 0.456165 & 0.476980 & 0.512701 \\
\cline { 2 - 5 } & -0.75 & 0.112881 & 0.172313 & 0.251607 \\
\cline { 2 - 5 } & -0.25 & 0.671978 & 0.681537 & 0.700609 \\
\cline { 2 - 5 } & -0.50 & 0.442028 & 0.461785 & 0.495907 \\
\hline \multirow{2}{*}{0.1} & -0.75 & 0.127332 & 0.181855 & 0.256264 \\
\cline { 2 - 5 } & -0.25 & 0.643513 & 0.652733 & 0.671247 \\
\cline { 2 - 5 } & -0.50 & 0.429897 & 0.448675 & 0.481448 \\
\hline
\end{tabular}


T. R. Mahapatra et al. / JAFM, Vol. 6, No. 1, pp. 121-129, 2013.

Table 4 Wall temperature $\theta(0)$ for the PHF case taking $\operatorname{Pr}=0.72, E_{c}=0.1, R=10.0$ and $L=1.0$

\begin{tabular}{|c|c|c|c|c|}
\hline$\varepsilon$ & $\alpha$ & $\mathrm{M}=0.0$ & $\mathrm{M}=0.5$ & $\mathrm{M}=1.0$ \\
\hline \multirow{4}{*}{0.0} & -0.25 & -1.836451 & -2.417161 & -4.883131 \\
\cline { 2 - 5 } & -0.50 & -0.475106 & -0.720959 & -1.480051 \\
\cline { 2 - 5 } & -0.75 & 0.294271 & -0.559534 & -1.139001 \\
\cline { 2 - 5 } & -0.25 & -1.56026 & -1.969695 & -3.316171 \\
\cline { 2 - 5 } & -0.50 & -0.464484 & -0.701216 & -1.414480 \\
\hline \multirow{3}{*}{0.1} & -0.75 & 0.196327 & -0.465441 & -1.121706 \\
\cline { 2 - 5 } & -0.25 & -1.369821 & -1.689931 & -2.643261 \\
\cline { 2 - 5 } & -0.50 & -0.455156 & -0.684199 & -1.361421 \\
\cline { 2 - 5 } & -0.75 & 0.143001 & & \\
\hline
\end{tabular}

\section{CONCLUSION}

In this paper, the MHD axisymmetric stagnation-point flow over a continuously shrinking sheet is investigated when the flow is permeated by a uniform

magnetic field normal to the surface. The symmetry line of the stagnation flow and that of the sheet are non-aligned and the effect of non-alignment is also studied. Numerically it is observed that the solution domain expands as the magnetic parameter $\$ \mathrm{M} \$$ increases. Flow reversal is observed near the sheet and the region of reverse cellular flow near the shrinking surface decreases with increase in M. In the heat transfer analysis, we have assumed that the thermal conductivity is a linear function of the temperature. Temperature profiles are obtained for two different types of heating processes, viz., PST and PHF cases for different values of the physical parameters. The effect of the magnetic parameter $\mathrm{M}$ is to decrease the temperature profile for both PST and PHF cases.

\section{ACKNOWLEDGMENT}

We thank the reviewer for his comments and suggestions which enabled us to make an improved presentation of the paper. The work of one of the authors (T.R.M) is supported under SAP (DRS PHASE II) program of UGC, New Delhi, India.

\section{REFERENCES}

Attia, H.A.. (2007) Axisymmetric stagnation point flow towards a stretching surface in the presence of a uniform magnetic field with heat generation, Tamkang. Journal of Science and Engineering, 10(1), 11-16.

Bansal, J.L. (1994) Magnetofluiddynamics of viscous fluid, Jaipur Publishing House, Jaipur, India.

Carragher, P. and Crane, L.J. (1982) Heat transfer on a continuous stretching sheet, Zeit. Angew. Math. Mech., $62,564-565$.

Chakrabarti, A. and Gupta, A.S. (1979) Hydromagnetic flow and heat transfer over a stretching sheet, Quarterly of Applied Mathematics, 37, 73-78.

Chamkha, A.J. and Ahmed, S.E. (2011) Similarity solution for unsteady MHD flow near a stagnation-point of a three dimensional porous body with heat and mass transfer, heat generation/absorption and chemical reaction, Journal of Applied Fluid Mechanics, 4(3), 8794.

Chiam, T.C. (1994) Stagnation-point flow towards a stretching plate, Journal of Physical Society of Japan, 63, 2443-2444.

Chiam, T.C. (1998) Heat transfer in a fluid with variable thermal conductivity over stretching sheet. Acta Mechanica, 129, 63-72.

Crane, L.J. (1970) Flow past a stretching plate, Zeit. Angew. Math. Phys., 21(4), 645-647.

Fang, T. (2008) Boundary layer flow over a shrinking sheet with power-law velocity, International Journal of Heat and Mass Transfer, 51(25-26), 5838-5843.

Fang, T. and Zhang, J. (2009) Closed-form exact solutions of MHD viscous flow over a shrinking sheet, Communication in Nonlinear Science and Numerical Simulation, 14, 2853-2857.

Goldstein S. (1965) On backward boundary layers and flow in converging passages, Journal of Fluid Mechanics, 21, 33-45.

Gupta, A.S.,and Gupta, P.S. (1977) Heat and mass transfer on a stretching sheet with suction and blowing, Canadian Journal of Chemical Engineering, 55, 744746.

Hiemenz K (1911), Die Grenzschicht in Einem in Dem Gleichformingen Flussigkeitsstrom Eingetauchten Gerade Kreiszlinder, Dingler Polytech Journal, 326, 321-410.

Homann, F. (1936) Der Einfluss grosser Zahigkeit bei der stromung um den Zylinder und um die Kugel', Zeit. Angew. Math. Phys., 16, 153-164.

Mahapatra, T.R. and Gupta, A.S. (2002) Heat transfer in stagnation-point flow towards a stretching sheet, Heat Mass Transfer, 38, 517-521. 
T. R. Mahapatra et al. / JAFM, Vol. 6, No. 1, pp. 121-129, 2013.

Mahapatra, T.R. and Gupta, A.S. (2003) Stagnation-point flow towards a stretching surface, Canadian Journal of Chemical Engineering, 81, 258-263.

Mahapatra, T.R. Nandy, S.K. and Gupta, A.S. (2011) Momentum and heat transfer in MHD stagnation-point flow over a shrinking sheet, Transaction to ASME, Journal of Applied Mechanics, 78,021015.

Miklavcic, M. and Wang, C.Y. (2006) Viscous flow due to a shrinking sheet, Quarterly of Applied Mathematics, 64(2), 283-290.

Pavlov, K.B. (1974) Magnetohydrodynamic flow of an incompressible viscous fluid caused by the deformation of a plane surface, Magnitnaya Gidrodinamika, 4, 146147.

Prasad, K.V., Pal D. and Datti, P.S. (2009) MHD power-law fluid flow and heat transfer over a non-isothermal stretching sheet, Communication in Nonlinear Science and Numerical Simulation, 14, 2178-2189.

Prasad, K.V. and Vajravelu, K. (2009) Heat transfer in MHD flow of a power-law fluid over a non-isothermal stretching sheet,, International Journal of Heat and Mass Transfer, 52, 4956-4965.
Rahimpour, M., Mohebpour, S.R., Kimiaeifar, A. and Bagheri, G.H. (2008) On the analytic solution of axisymmetric stagnation flow towards a shrinking sheet, International Journal of Mechanics, 1(2), 1-10.

Savvas, T.A, Markatos, N.C., Papaspyrides, C.D. (1994). On the flow of non-Newtonian polymer solutions, Applied Mathematical Model, 18, 14-22.

Sharma, P.R. and Singh, G. (2009). Effects of Variable Thermal Conductivity and Heat Source / Sink on MHD Flow Near a Stagnation Point on a Linearly Stretching Sheet, Journal of Applied Fluid Mechanics, 2, 13-21.

Shercliff, J.A. (1965). A text book of Magnetohydrodynamics, Pergamon Press, Oxford.

Wang, C.Y. (1984). The three dimensional flow due to a stretching flat surface, Physics of Fluids, 27, 19151917.

Wang, C.Y. (2008). Stagnation flow towards a shrinking sheet, International Journal of Nonlinear Mechanics, $43,377-382$ 